In Situ

Revue des patrimoines
In Situ

Revue des patrimoines

40 | 2019

Lits historiques. Première anthologie des lits européens du XVe au XIXe siècle

\title{
Le lit royal à l'aube de la Renaissance
}

Royal Beds of the Early Renaissance

\section{Caroline Vrand}

\section{(2) OpenEdition}

Journals

Édition électronique

URL : http://journals.openedition.org/insitu/22676

DOI : 10.4000/insitu.22676

ISSN : 1630-7305

Éditeur

Ministère de la Culture

Référence électronique

Caroline Vrand, «Le lit royal à l'aube de la Renaissance », In Situ [En ligne], 40 | 2019, mis en ligne le 19 septembre 2019, consulté le 14 novembre 2019. URL : http://journals.openedition.org/insitu/22676 ; DOI : 10.4000/insitu.22676

Ce document a été généré automatiquement le 14 novembre 2019.

\section{(c) $($ ) $\odot$}

In Situ Revues des patrimoines est mis à disposition selon les termes de la licence Creative Commons Attribution - Pas d'Utilisation Commerciale - Pas de Modification 4.0 International. 


\title{
Le lit royal à l'aube de la Renaissance
}

Royal Beds of the Early Renaissance

\author{
Caroline Vrand
}

1 Vers 1500, dans les résidences royales de Charles VIII (1483-1498) et de Louis XII (1498-1515), les lits constituaient une part primordiale de l'aménagement des logis royaux. Pourtant, il ne reste aujourd'hui rien de ces nombreux lits et des textiles qui les recouvraient ${ }^{1}$. Les sources écrites, tout particulièrement les inventaires et les comptes royaux, viennent en revanche compenser cette absence de vestiges matériels. Ainsi, la comptabilité de Charles VIII et de Louis XII, et plus encore, les inventaires d'Anne de Bretagne (1491-1514), épouse successivement des deux rois de France, fournissent quantité d'informations sur les lits dressés dans les logis royaux ${ }^{2}$. L'étude de ces documents permet d'appréhender l'aspect matériel des lits - les différents éléments qui les composent, leurs matières, leurs couleurs - et aussi de mesurer toute l'importance de cet objet au sein de l'aménagement des châteaux et, plus largement, des collections royales.

\section{Le châlit et le lit : la suprématie des étoffes}

2 La langue de l'époque distinguait la structure de menuiserie, le châlit, de sa garniture textile, le lit. Le châlit n'était pas la partie importante de l'objet, au point d'ailleurs de retenir rarement l'attention des rédacteurs des inventaires car les regards se concentraient sur les étoffes, de laine et de soie, qui recouvraient le plus souvent totalement les parties en bois.

Le bois de lit présentait une forme à peu près similaire à celle que nous lui connaissons aujourd'hui : un grand cadre monté sur des pieds, avec un fond garni de cordes ou de sangles. Une paillasse, grand coussin rempli de paille, constituait une sorte de premier matelas. Par-dessus se trouvaient le matelas, coussin en coton ou laine, et parfois le doublet, sorte de courtepointe de toile abondamment garnie de laine ou de plumes et 
étendue entre le matelas et le drap pour rendre le coucher plus souple et plus agréable. La présence d'une tête de lit en bois, appelée tresdos, n'était pas systématique et un matelas pouvait la remplacer. D'après les inventaires, il reste difficile de distinguer différents types de châlit. Les documents mentionnent bien des "lits de camp », dont on pourrait croire qu'il s'agit de lits peu encombrants, particulièrement adaptés pour les déplacements de la Cour. Pourtant, il apparaît que ces lits de camp meublaient les logis royaux lors des événements les plus solennels, comme c'est le cas dans les chambres attribuées à l'archiduc d'Autriche Philippe le Beau et à sa femme Isabelle de Castille lorsqu'ils séjournent à Blois en $1501^{3}$. Indistinctement décrits comme des lits de camp, ces deux lits n'offraient cependant pas la même structure, puisque celui de l'archiduchesse était à quenouilles, c'est-à-dire avec une colonne à chacun des quatre angles. Plusieurs autres occurrences confirment que ces lits de camp étaient loin de n'être que d'humbles meubles dont la facture simple convenait au déplacement: dans sa chambre du Plessis-lès-Tours ${ }^{4}$, Charles VIII dort dans un lit de camp recouvert de drap d'or ${ }^{5}$, en 1492 Anne de Bretagne donne naissance à Charles-Orland dans un lit de camp lui aussi tendu de drap d'or ${ }^{6}$ et c'est encore un lit de camp somptueusement décoré qu'Isabelle d'Este offre à la reine Anne vers $1501^{7}$.

Si la structure en bois peut, de façon exceptionnelle, être ornée, ce sont néanmoins les tissus qui la recouvrent qui attirent toute l'attention ${ }^{8}$. Cette garniture textile se compose de plusieurs éléments, dont la pièce la plus emblématique est le ciel, tendu horizontalement au-dessus du lit et maintenu au châlit grâce à des cordes, fixées aux poutres de la pièce, qui peuvent être teintes de la couleur des étoffes (fig. 1)99.

Figure 1

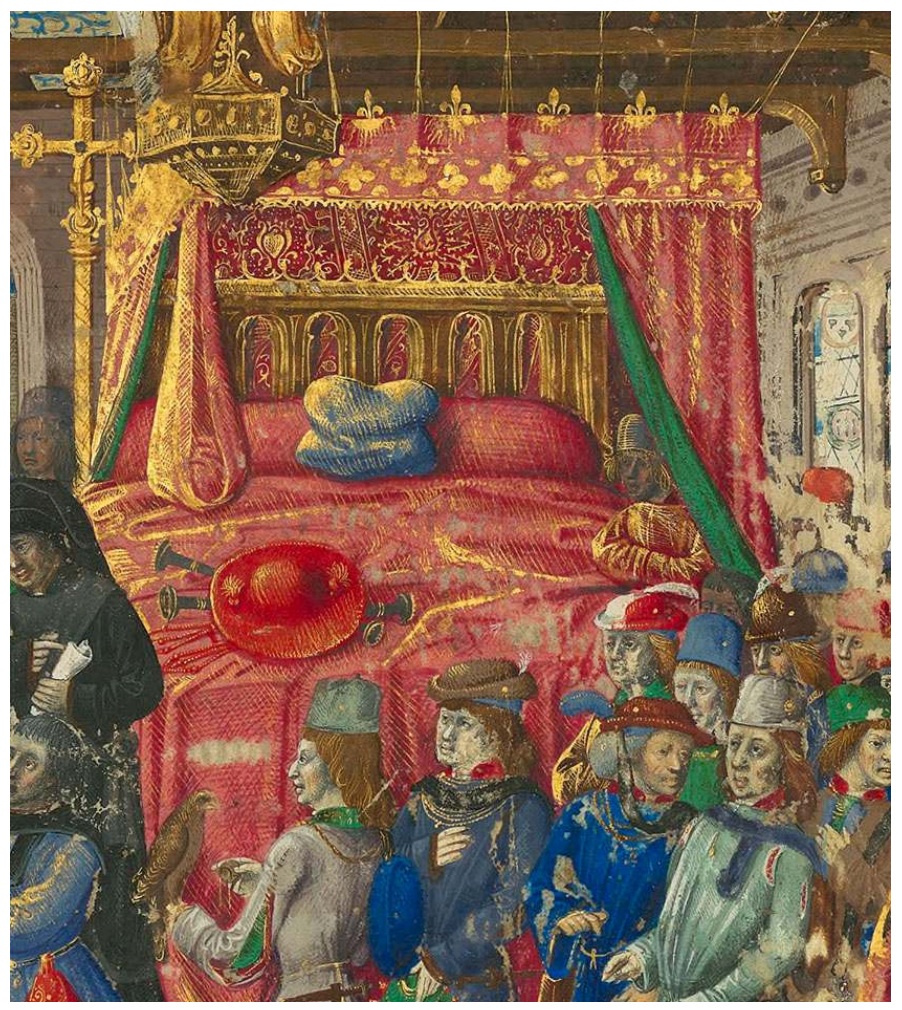

Maître du cardinal de Bourbon, Le cardinal de Bourbon recevant l'ouvrage de la vie de saint Louis, dans Le Livre des faiz Monseigneur saint Loys. BnF, ms fr. 2829, fo 3 (vue partielle).

(c) BnF. 
En 1488, dans la chambre de Charles VIII au Plessis-lès-Tours, soixante-douze aunes de corde blanche et rouge servent ainsi à tendre le ciel et les rideaux ${ }^{10}$. Lorsque le ciel prend une forme conique, il est appelé pavillon. Même les berceaux princiers avaient parfois un pavillon, comme celui de Claude de France, fille de Louis XII et d'Anne de Bretagne, lors de la réception de l'archiduc d'Autriche à Blois ${ }^{11}$. La tête du lit recevait, quant à elle, un tissu appelé dossier, dossel ou muraille. Autour du lit, de longs rideaux, aussi appelés courtines, tombaient jusqu'au sol. Le plus souvent au nombre de trois, parfois quatre voire six, ils s'accrochaient sur de petits anneaux courant sur une tringle (fig. 2). Soigneusement fermés durant la nuit, ils se relevaient durant la journée. Un même lit pouvait comporter deux types de rideaux différents. Celui dans lequel dort Charles VIII au Plessis-lès-Tours en 1492 dispose ainsi d'un jeu en drap d'or, assorti au ciel, et d'un autre en taffetas blanc, sorte de voilages « pour servir ou lieu de deux autres rideaux de drap d'or quant lesd. rideaulx sont levez sur le ciel afin que led. seigneur puisse mieulx veoir la clarté ${ }^{12}$. À la jonction du ciel et des rideaux, les pentes ou gouttières, bandes de tissu d'une vingtaine de centimètres de large, faisaient le tour $\mathrm{du}$ ciel et servaient à cacher les tringles et autres systèmes de fixation. Enfin, couvertures et courtepointes complétaient l'ensemble. Garnies de plume ou de laine et doublées de coton ou de soie pour les plus riches, elles enveloppaient complètement le châlit, tombant jusqu’à terre.

Figure 2

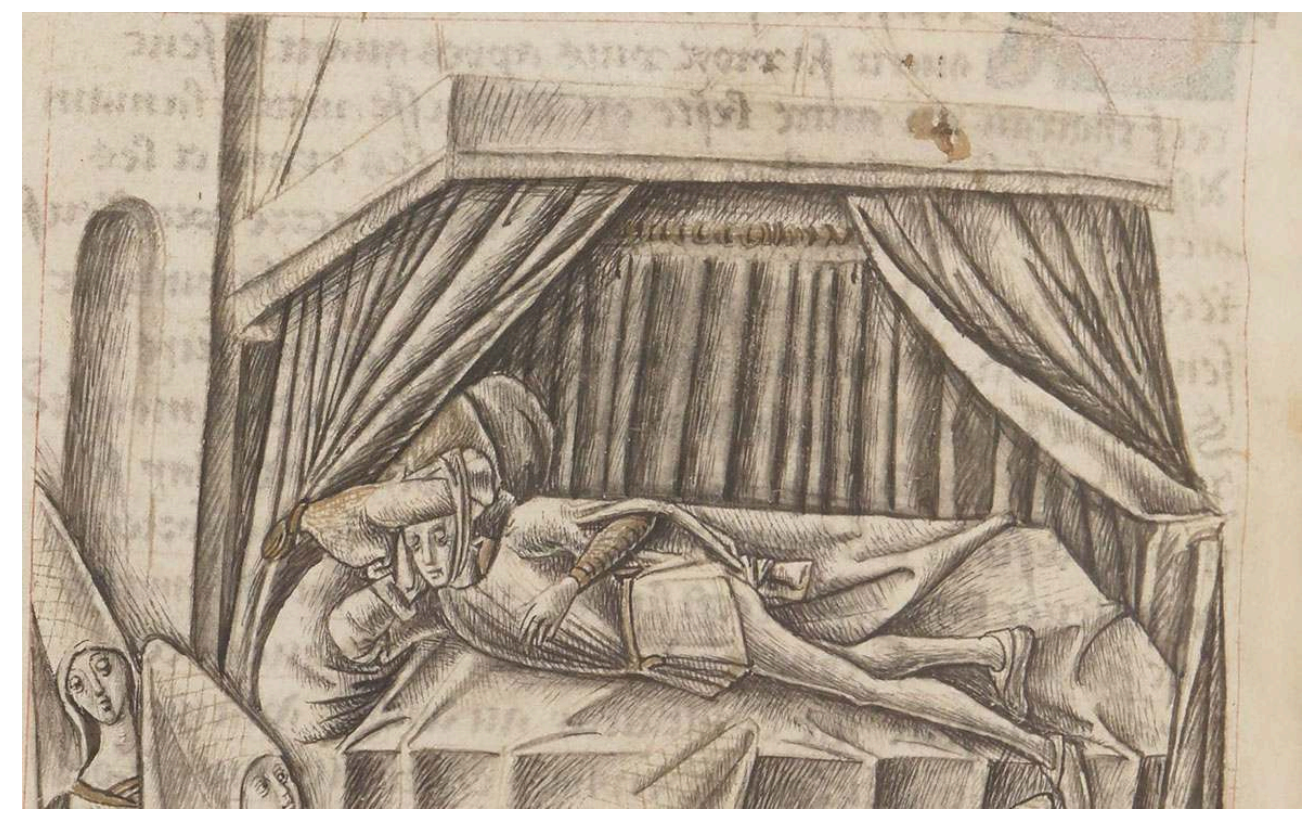

Filostrato de Boccace, milieu du xve siècle. BnF, ms fr. 25528, fo $85 v^{\circ}$ (vue partielle)

(C) BnF.

6 Le recours à ces diverses pièces textiles découle en premier lieu du besoin de se protéger du froid et des courants d'air : le dossier isole de l'humidité du mur, le ciel, les rideaux et les courtines conservent la chaleur. Néanmoins, au-delà de ces considérations pratiques, les princes firent de ces éléments textiles de véritables signes extérieurs de leur richesse, expressions de leur rang. Ciel, dossier et rideaux constituaient le plus souvent un ensemble assorti, que pouvaient aussi compléter la couverture, les carreaux - coussins pour s'asseoir -, le banchier - textile recouvrant les bancs - et des tentures 
murales. Les matières employées pour la confection de ces textiles dépendent naturellement du rang de leur propriétaire et, à la Cour royale, la soie domine dans toutes ses armures - damas, taffetas, velours ou même velours broché d'or, étoffe la plus précieuse de toutes. Si la plupart des garnitures textiles de lit étaient des soieries, le recours à la tapisserie était aussi possible. Il faut ici admettre que l'identification des techniques de tissage d'après les inventaires n'est pas toujours aisée, d'autant que le terme de "tapisserie " se voit employé dans un sens très générique et peut regrouper aussi bien les soieries, les broderies que les tapisseries à proprement parler. Malgré tout, il nous paraît possible de reconnaître dans certains cas de véritables ouvrages de tapisserie employés comme parure de lit. Les rédacteurs des inventaires s'efforcent d'ailleurs de distinguer les tapisseries « de chambre ", destinées à décorer les lits et les murs de la chambre, de celles "de salle", appelées à recouvrir les murs de la grande salle. Les erreurs d'appréciation ne sont pas impossibles. Dans un registre de 1498, la mention de "tapisserie de chambre " avec une scène de chasse a été corrigée par un relecteur, qui prit soin de remplacer le terme de «chambre " par celui de « salle $»^{13}$. Il apparait en fait que, dans les chambres des logis royaux, les tapisseries qui ornaient les lits pouvaient appartenir à la même suite que celles tendues aux murs. Une même iconographie se déployait alors dans l'ensemble de la pièce, voire du logis, si l'on considère que le décor pouvait s'étendre aux murs de la salle. Parmi les grands cycles de tapisserie en possession d'Anne de Bretagne, plusieurs comportaient ainsi des éléments de parure de lit. C'est le cas de «la tappisserie appellée Guyenne », qui devait relater la reconquête de l'Aquitaine par les troupes de Charles VII, et qui comportait six pièces dont un ciel, un tresdos et une couverture, les autres éléments étant certainement destinés à rejoindre les murs. Mentionnons également une Histoire d'Alexandre, particulièrement précieuse puisque rehaussée de fils d'or, répartie en huit pièces, dont un ciel, un dossier, une couverture de lit et une couverture de couchette ${ }^{14}$. Néanmoins, dans les chambres, la préférence en matière d'iconographie semble aller aux scènes évoquant la vie quotidienne et aux représentations mettant à l'honneur la nature. Anne de Bretagne possédait ainsi plusieurs tentures de chasses avec ciels, dossiers, couvertures, gouttières, banchiers et coussins et la plupart des millefleurs - désignées "verdure" ou "menue verdure" - conservées au château d'Amboise en 1494 comportaient au moins un élément de parure de lit, le plus souvent un ciel, des rideaux ou une couverture ${ }^{15}$.

\section{Le lit au cœur du logis royal : quelques commandes emblématiques}

7 À une époque où les meubles, au sens actuel du terme, restaient rares, le châlit et ses textiles constituaient les pièces essentielles de l'aménagement des logis royaux. Les éléments de parure de lit abondent dans les inventaires, et les mentions concernant les châlits, bien que peu fréquentes, n'en indiquent pas moins leur nombre important au sein des châteaux. Un inventaire de décembre 1500 en signale ainsi trente-six à Amboise, dont quatre lits de camp ${ }^{16}$. Le nombre de châlits dépassait donc largement celui des chambres du château. Ils pouvaient en effet se trouver également dans les chambres de retrait ou les garde-robes et une même pièce en abritait souvent plusieurs. Pour autant, les lits les plus soignés prenaient place dans les chambres du roi et de la 
reine. Charles VIII comme Anne de Bretagne ont ainsi tous deux passé de prestigieuses commandes qui rendent bien compte de la portée ostentatoire des parures de lit.

Concernant Charles VIII, nous souhaitons ici nous attacher aux commandes que ce dernier effectua pour le château du Plessis-lès-Tours, qui constitue le premier grand chantier d'envergure du roi, quelques années avant celui d'Amboise ${ }^{17}$. Ces travaux sont peu documentés et restent mal connus des historiens de l'architecture, mais le compte de l'hôtel du roi des années 1489-1490 mentionne « le logis neuf du Plessis », la " garde robe neuve du roi », la «garde robe que le roi a fait faire dans l'hotel du Plessis » et la "chambre haulte neufve que le roi a fait faire au Plessis", autant d'indices qui permettent de conclure que Charles VIII fit construire un nouveau logis, au premier étage ${ }^{18}$. Comme pour le château d'Amboise à partir de 1493 , le roi ne se contenta pas d'ériger les murs du logis, il s'attacha aussi à en orner les pièces avec soin. C'est à Raymond de Dezest, son valet de chambre et tailleur, qu'incomba «la charge de l'emmenagement du logis neuf du Plessis ${ }^{19}$. De mai à juin 1491, le roi se fit livrer une importante quantité de velours pour la réalisation de fastueux ensembles d'apparat. La plupart de ces tissus étaient destinés à ses lits : ainsi, un pavillon de taffetas violet pour un de ses lits de camp; deux ensembles "my-parti de rouge et de tanné ", l'un de damas et l'autre de taffetas, composés chacun d'un ciel, d'une couverture, d'un dossier, de trois rideaux et deux pentes pour deux autres de ses lits de camp; deux ensembles similaires de taffetas tanné - couleur brun roux - et rouge, l'un pour la couchette de la nouvelle garde-robe et l'autre pour le lit de sa chambre ${ }^{20}$. Quelques mois plus tard, Charles VIII passa à nouveau une importante commande dont on peut penser qu'elle participe de l'aménagement de la chambre de son nouveau logis tourangeau. En novembre 1491, il confia en effet plusieurs aunes de damas noir au brodeur Hesterlin de Dricq afin qu'il réalise six cent quatre-vingt-sept «lettres de SS romaines a cordelieres par dedans de la grandeur d'un pié en carré pour icelles mectre et asseoir,

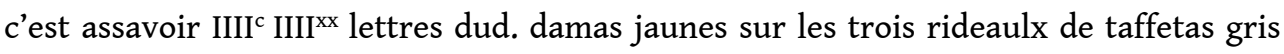
dont cy dessus est fait mention et IIII ${ }^{c}$ VII pareilles lettres faictes dud. damas gris sur lesd. ciel, dociel et pentes de satin jaune ». L'année suivante, en mars 1492, Raimond de Dezet se vit chargé de la confection d'un nouvel ensemble de damas jaune et gris, composé d'un ciel «my parti des deux couleurs pour servir a tendre sur le lit ou couche led. Seigneur ", d'un dossier " pour servir aud. ciel », de quatre pentes du ciel, « les deux des deux coustez [...] et les deux des deux boutz » et de trois rideaux « pour servir aud. lit ». Les matières employées pour ces différents lits, le damas et le taffetas, indiquent qu'ils relevaient vraisemblablement d'un usage ordinaire, dans la mesure où il s'agit là d'armures de soie moins luxueuses que le velours ou le drap d'or, privilégiés pour les réceptions et grands événements de la vie de Cour. Pour autant, ces commandes, notamment celle de novembre 1491, montrent comment le lit s'impose comme un support de prédilection de l'emblématique royale : le roi appose son chiffre, le double $\mathrm{S}$, qu'il associe à la cordelière, devise des ducs de Bretagne, alors même qu'il administre la Bretagne depuis sa prise de possession du château de Nantes en mars 1491 et qu'il se considère comme le successeur légitime du duc François II ${ }^{21}$.

Dès son arrivée en Val de Loire, Anne de Bretagne entend elle aussi marquer de son empreinte l'aménagement de ses logis, ce qui passe en premier lieu par la commande d'étoffes de lit. En septembre 1492, alors qu'elle n'est reine de France que depuis quelques mois, elle sollicite le brodeur du roi, Guillaume Martin, afin qu'il réalise un patron pour un ciel de lit de damas mi-parti jaune et rouge semé de cordelières de 
velours noir ${ }^{22}$. Cette demande est exceptionnelle à plus d'un titre. Le fait que la reine fasse appel à un brodeur afin de concevoir un patron témoigne de l'importance accordée à cette réalisation par la commanditaire. Cela révèle sa volonté de s'assurer, avant l'exécution définitive du ciel, de sa qualité et de se laisser la possibilité, si besoin, d'intervenir et de demander des modifications. De surcroît, Anne veille à y faire apposer l'emblème des ducs de Bretagne, la cordelière. Cette commande de la reine peut apparaître comme une réponse à celle du roi passée en mars de la même année. Les couleurs choisies, mi-parti jaune et rouge, peuvent surprendre, puisqu'elles sont avant tout connues pour être celles de Louis XII. Il faut pourtant admettre, au vu de cette commande, qu'elles furent aussi choisies par Anne de Bretagne, dès sa première année à la Cour de France. Quant à la présence de la cordelière, elle est pleinement porteuse de sens. Anne de Bretagne, conformément au traité établi à l'occasion de son mariage avec Charles VIII, vient de perdre le titre de duchesse de Bretagne, mais elle choisit d'arborer sur son lit, au cœur du logis royal, cet emblème ducal, dont l'usage s'était particulièrement diffusé à la Cour de son père le duc François II ${ }^{23}$.

Quelques années plus tard, en juin 1496, Anne de Bretagne passa une nouvelle commande prestigieuse, pour le lit de son logis tourangeau. Il s'agit cette fois non pas d'étoffes de soie mais de soubassements en cuir. Pour ce faire, elle fit appel à un "paintre ytalien que led. seigneur avoit fait venir de Napples», qui résidait alors «a [blanc] pres Amboise ». À sa demande, l'artiste se rendit à Tours pour « faire et paindre les soubzbassemens d'un lit de camp faiz de groz cuir couroyé en alun [...]». On apprend ailleurs qu'il s'agit d'un "grand cuir de bueuf blanc " ${ }^{24}$. L'artiste napolitain en question est très probablement Guido Mazzoni, sculpteur originaire de Modène, qui s'installa à Naples en 1488 après avoir été appelé par le duc de Calabre, Alphonse II, et qui compte parmi les artistes que Charles VIII ramena avec lui de son expédition italienne ${ }^{25}$. D'après un document comptable de janvier 1498, il est le seul peintre parmi la vingtaine d'Italiens entrés au service de Charles VIII et son installation à Amboise est attestée à partir de $1496^{26}$. Ses réalisations à la cour de France restent mal connues et cette intervention de juin 1496 sur les soubassements en cuir du lit d'Anne de Bretagne apparaît comme la première trace de l'activité de Guido Mazzoni en France pour la Cour royale ${ }^{27}$. Il n'est pas anodin qu'Anne de Bretagne sollicite cet artiste, à n'en pas douter auréolé du prestige de son origine, à un moment où elle entend prendre pleinement possession de son logis au Plessis-lès-Tours. En effet, à partir de la fin de l'année 1493, la reine investit, au Plessis, le logis neuf construit par Charles VIII, situé à l'étage noble, certainement à la faveur d'une installation plus durable du roi au château d'Amboise ${ }^{28}$. La reine effectua alors plusieurs travaux d'aménagement, aussi bien dans les chambres de ses demoiselles que dans sa propre chambre, sa garde-robe ou encore sa salle. Tout porte à croire qu'Anne de Bretagne avait pour ambition de faire de Tours l'équivalent de ce qu'était Amboise pour le roi, à savoir un château soigneusement meublé et au sein duquel elle tenait sa cour. Le choix de soubassements en cuir révèle certainement sa volonté d'afficher une certaine distinction et aussi une certaine modernité inspirée par les modes italiennes. Vers 1500 en effet, les décors en cuir, qu'ils soient peints ou dorés, sont rares à la cour de France et, nous l'avons vu, les matières privilégiées pour les garnitures de lit sont les étoffes de soie ou les tentures de laine. Si l'emploi de décors en cuir dans les intérieurs royaux et princiers, et notamment pour les lits, est attesté vers 1400 , comme le montrent les mentions d'éléments en cuir "d'Aragon » ou «de Hongrie » dans les inventaires de Charles V (1380), les comptes d'Isabeau de Bavière (1393) ou encore du duc d'Orléans (1427) ${ }^{29}$, les 
inventaires royaux de la fin du $\mathrm{xv}^{\mathrm{e}}$ siècle montrent que cet usage avait largement décliné. Du vivant d'Anne de Bretagne, peu d'artistes devaient maîtriser la technique de la peinture sur cuir ${ }^{30}$. Cela pourrait aussi expliquer pourquoi la reine eut recours à un peintre italien. Par son séjour à Naples, Guido Mazzoni avait de surcroît pu se familiariser avec le travail du cuir, les liens dynastiques entre le royaume de Naples et ceux de Castille et d'Aragon ayant certainement favorisé l'importation des cuirs espagnols, particulièrement réputés ${ }^{31}$.

\section{Le lit comme cadeau diplomatique}

11 Non seulement le lit fait l'objet d'importantes commandes, mais il constitue aussi un cadeau diplomatique apprécié, preuve supplémentaire de son caractère prééminent au sein des collections royales et princières. En décembre 1491, l'un des premiers présents que Charles VIII fit parvenir à Anne de Bretagne lorsque celle-ci devint reine de France, au moment de son entrée dans le royaume de France, est justement un lit de camp orné de précieuses étoffes de velours broché d'or ${ }^{32}$. Quelques années plus tard, Louis XII offre à son tour des textiles de lit à son épouse. Il s'agit de deux ensembles rapportés de son expédition milanaise, dont il revient en novembre 1499. Anne de Bretagne les entrepose d'abord dans son château de Nantes, en janvier 1500, puis les fait envoyer à Blois $^{33}$. Le premier de ces lits, de satin cramoisi, se compose d'un ciel, d'un dossier, d'une couverture et de trois gouttières. Chacune des pièces porte cinq lions brodés « faiz de perle ", coiffés d'un heaume et tenant entre leurs pattes un écusson duquel pendent deux sceaux. Ces précieuses étoffes brodées devaient être réservées aux festivités les plus solennelles. Ainsi concourent-elles à la décoration du château de Blois lors de la réception de l'archiduc Philippe le Beau et de sa femme Isabelle de Castille en décembre 1501. Elles retiennent d'ailleurs toute l'attention du chroniqueur qui relate l'événement et qui précise que ce lit « estoit fort riche à voir, et disoit-on qu'il avoit bien cousté quarante à cinquante mille ducats ». Trente ans plus tard, ces soieries continuent d'être exposées lors des rencontres diplomatiques, puisque le ciel est tendu en 1532 pour l'entrevue qui réunit, à Bologne, François $\mathrm{I}^{\mathrm{er}}$ et le roi d'Angleterre Henri VIII. Le second ensemble rapporté de Milan par Louis XII comprend trois pièces "sur satin cramoisy et satin blanc escartellee, a ouvraiges de broderie a ondes et volleries ", " semé d'oiseaux, de riviere ». Comme le ciel précédent, il passe ensuite dans les collections de François $\mathrm{I}^{\mathrm{er}}$. Que ces deux parures lit soient brodées est, il faut le souligner, tout à fait exceptionnel. $A u \mathrm{Xv}^{\mathrm{e}}$ siècle, la plupart des textiles de lit sont en étoffe de soie ou en tapisserie, rarement en ouvrage de broderie. De façon générale, les éléments brodés restent exceptionnels dans l'ameublement et se limitent le plus souvent à l'application des armes et emblèmes. Les deux ciels milanais, où des broderies d'or rehaussées de perles constituent l'essentiel du décor, font ainsi exception au sein des collections royales ${ }^{34}$. Rapportés de l'expédition milanaise du roi, il est probable qu'ils avaient été confectionnés dans les ateliers de la capitale ducale. Milan était du reste célèbre, depuis le XIII ${ }^{\mathrm{e}}$ siècle, pour sa fabrication d'étoffes de soie, son or filé et sa broderii $^{35}$. Louis XII semble en tout cas avoir particulièrement apprécié le savoir-faire des artisans milanais puisqu'en 1507-1508, il acheta à des « marchans et gens de mestier de lad. ville de Millan » plus de mille sept cents livres de «draps de soye, toille d'or et d'argent, fil d'or d'argent et de soye, autres parties de sellerie et broderye ${ }^{36}$. 
12 La pratique du don de lits ne se limite pas à la cour de France et les inventaires d'Anne de Bretagne apportent également un éclairage sur la our mantouane. Frédéric I ${ }^{\text {er }}$ de Gonzague, marquis de Mantoue de 1478 à 1484, envoya ainsi au roi de France un pavillon de drap d'or, encore soigneusement conservé à Blois en 1507. Quelques années plus tard, vers 1500, c'est au tour d'Isabelle d'Este, épouse du marquis François II de Gonzague, d'adresser à Anne de Bretagne un lit somptueux. À une période où la cour de France et celle de Mantoue cherchent à renforcer leurs liens diplomatiques, les échanges entre les deux souveraines se multiplient et passent aussi par l'envoi de cadeaux $^{37}$. La marquise de Mantoue avait d'ailleurs pour habitude d'offrir aux princesses des cours européennes divers présents reflétant son goût prononcé pour les arts, que ce soient des tableaux ou des objets d'art. Elle fit ainsi parvenir à Anne de Bretagne un tableau de Lorenzo Costa (1460-1535), artiste qui, à la mort d'Andrea Mantegna en 1506, lui succéda comme premier peintre de cour. Le lit que reçoit Anne de Bretagne vers 1500 est un ensemble complet. Il comprend le châlit, soigneusement sculpté et doré, semé de fleurs de lys et d'hermines et reposant sur six pieds en forme de pattes de lion, auquel s'ajoute la garniture textile, de précieuses étoffes aux armes de la reine et à son emblème, la cordelière. Isabelle d'Este veilla également à accompagner le tout d'un grand « tapis velu » pour recouvrir le sol de la pièce où le lit serait monté et de deux très grands coffres afin d'y entreposer l'ensemble de ses éléments lorsqu'il serait « détendu $»^{38}$.

13 Les lits, et tout particulièrement leurs garnitures textiles, sont abondamment mentionnés et décrits dans la comptabilité et les inventaires royaux de Charles VIII, Louis XII et Anne de Bretagne, ce qui donne la mesure de leur importance et de leur place au sein des collections royales. Une étude plus approfondie de ces documents permet de surcroît de mettre en lumière combien le lit constitue un élément pivot non seulement de la chambre, mais du logis royal dans son ensemble. Le réaménagement d'un château, la construction d'une nouvelle aile s'accompagnent presque nécessairement de la commande de nouvelles parures de lit et, en la matière, les commandes royales de premier ordre sont, nous l'avons vu, nombreuses. Pour leurs lits, le roi et la reine sollicitent des artistes de renom, supervisent la confection, veillent au choix des couleurs et à la mise en valeur de leur emblématique. Les représentations enluminées d'Anne de Bretagne la montrant, accompagnée de ses dames, dans une chambre où trône le lit, sont à cet égard révélatrices de la dimension véritablement ostentatoire de ce meuble ${ }^{39}$. Indéniablement, le lit royal participe de l'affirmation personnelle du souverain et de la mise en scène de son pouvoir.

\section{NOTES}

1. - Sur l'ampleur de ces pertes, voir CRÉPIN-LEBLOND, Thierry. «Le mobilier royal de la Renaissance française: enquête sur une disparition». Dans THIRION, Jacques et CRÉPINLEBLOND, Thierry (dir.). Parures d'Or et de Pourpre: le mobilier à la cour des Valois. Cat. exp., Blois, château de Blois, 15 juin-30 septembre 2002. Paris/Blois: Somogy éd. d'art/Château de Blois, 2002, p. 19-39. 
2. - Les comptes de l'hôtel et de la trésorerie de Charles VIII et Louis XII sont conservés aux Archives nationales (AN). Les inventaires d'Anne de Bretagne sont conservés à la Bibliothèque nationale de France (BnF, ms fr. 22335). Nous avons mené une étude approfondie de ces inventaires dans notre thèse de doctorat, à laquelle nous renvoyons pour plus d'informations sur leur nature, leur apport et aussi pour leur édition intégrale : VRAND, Caroline. Les Collections d'art d'Anne de Bretagne. Au rythme de la vie de cour. Thèse d'histoire de l'art. Paris : université Paris-1Panthéon-Sorbonne, 2016 (à paraitre). Pour les inventaires d'Anne de Bretagne, voir aussi leur édition partielle dans LE ROUX de LINCY, Antoine. Vie de la reine Anne de Bretagne: femme des rois de France Charles VIII et Louis XII, suivie de lettres inédites et de documents originaux. Paris : L. Curmer, 1860-1861.

3. - BnF, ms fr. 22335, p. 6. Pour l'étude de cette réception, voir aussi CHATENET, Monique et GIRAULT, Pierre-Gilles. Fastes de cour : les enjeux d'un voyage princier à Blois en 1501. Rennes: PUR, 2010.

4. - Le château du Plessis-lès-Tours se situe dans la commune de La Riche, en Indre-et-Loire.

5. - AN, KK 70, fo 267.

6. - BnF, ms fr. 22335, p. 15-18.

7. - Voir infra pour ce cadeau d'Isabelle d'Este.

8. - Sur l'importance des textiles dans l'ameublement royal, voir THIRION, Jacques et CRÉPINLEBLOND, Thierry (dir.). Op. cit.

9. - La date d'apparition du ciel varie du XIII ${ }^{\mathrm{e}}$ siècle au XIV ${ }^{\mathrm{e}}$ siècle selon les historiens. Voir THIRION, Jacques. Le Mobilier du Moyen Âge et de la Renaissance en France. Dijon : Faton, 1998, p. 55 et MANE, Perrine. « Le lit et ses tentures d'après l'iconographie du XIII ${ }^{\mathrm{e}}$ au XV $\mathrm{XV}^{\mathrm{e}}$ siècle ». Mélanges de l'École française de Rome, 1999-1, 111-1, « Tentures médiévales dans le monde occidental et araboislamique ». Actes de la journée d'étude de Lyon, 16 mars 1994, éd. Françoise Piponnier, p. 393-418, ici p. 396.

10. - AN, KK 70, fo 267.

11. - Voir la relation de l'événement publiée dans GODEFROY, Théodore. Le Cérémonial de France, ou Description des cérémonies, rang et séances observées aux couronnemens, entrées et enterremens des Roys et Roynes de France. Paris : A. Pacard, 1619, t. II, p. 713 sqq.

12. - AN, KK 72, fo 49.

13. - BnF, ms fr. 22335, p. 12.

14. - BnF, ms fr. 22335, p. 152.

15. - BnF, ms fr. 22335, p. 12-33 et 155-157.

16. - BnF, ms fr. 22335, p. 187.

17. - Pour le chantier d'Amboise, nous renvoyons aux travaux d'Évelyne Thomas (« Les logis royaux d'Amboise ». Revue de l'Art, 1993, 100, p. 44-57) et à sa contribution dans ce numéro: THOMAS, Évelyne. «Les lits royaux d'Amboise sous Charles VIII et Anne de Bretagne : étoffes, couleurs et décor ", In Situ [En ligne], 40 |2019, mis en ligne le 18 septembre 2019, consulté le 30 septembre 2019. URL : http://journals.openedition.org/insitu/23834. Pour les travaux menés par Charles VIII au Plessis-lès-Tours, la bibliographie est pour l'heure limitée. Voir notamment CHEVALIER, Casimir et QUINCARLET, Edmond. « Le couvent de saint François de Paule au Plessislès-Tours (1490-1498) ». Mémoires de la Société archéologique de Touraine, 1873, t. XXIII, p. 123-140 ; SALAMAGNE, Alain. «Le Plessis et les résidences royales en Touraine à l'avènement de Louis XI ». Bulletin de la Société archéologique de Touraine, 2014, t. LX, p. 173-184.

18. - AN, KK 71, fo $23,81 v^{\circ}, 85$ et 148.

19. - AN, KK 71, fo 23.

20. - AN, KK 71, fo $72 \mathrm{v}^{\circ}, 82,84 \mathrm{v}^{\circ}$.

21. - Sur la guerre qui oppose le duché de Bretagne et le royaume de France et sur le statut de la Bretagne, voir LEGUAY, Jean-Pierre et MARTIN, Hervé. Fastes et malheurs de la Bretagne ducale, 1213-1532. Rennes : Ouest-France, 1982. 
22. - AN, KK 530-6. Le fait que la reine fasse appel à un brodeur pour la réalisation du patron de son ciel de lit corrobore l'affirmation de Louis de Farcy pour qui le brodeur conserve dans ses attributions, encore au xve siècle, l'exécution des lits, alors même que les ouvrages de broderie sont de plus en plus concurrencés par les tapisseries et les étoffes de soie (FARCY, Louis de. La Broderie du XI siècle à nos jours. Angers : Belhomme, 1890-1919, p. 94).

23. - Contrairement à ce qui a pu être avancé, Anne de Bretagne n'abandonne en effet pas l'usage de la cordelière durant son mariage avec Charles VIII. Voir par exemple l'étude suivante, qui reste déterminante par ailleurs : HABLOT, Laurent. « Pour en finir - ou pour commencer ! - avec l'ordre de la Cordelière ». Dans LEPAGE, Dominique (éd.). Pour en finir avec Anne de Bretagne? Actes de la journée d'étude organisée aux Archives départementales de Loire-Atlantique le 25 mai 2002. Nantes : Archives départementales de Loire-Atlantique, conseil général de Loire-Atlantique, 2004, p. 47-62.

24. - AN, KK 85, fo $81 v^{\circ}, 95$ et $95 v^{\circ}$. L'emploi de l'alun, qui provoque le blanchiment, permettait de traiter les peaux de bovidés et de les transformer en cuir. Sur cette technique, voir HALASZ CSIBA , Eva. « Le cuir de Hongrie en France entre les XIv et XvIII e siècles. Histoire et problématique d'un transfert technique basé sur l'usage de l'alun », et CHAHINE, Claire. «L'utilisation de l'alun dans la transformation de la peau en cuir ». Dans BORGARD, Philippe, BRUN, Jean-Pierre et PICON, Maurice (dir.). L'Alun de Méditerranée. Naples/Aix-en-Provence: Centre Jean Bérard/Centre Camille Jullian, 2005, p. 311-322 et p. 299-309.

25. - On lui doit notamment le tombeau de Charles VIII à Saint-Denis (détruit).

26. - MONTAIGLON, Anatole de. "États des gages des ouvriers italiens employés par Charles VIII ». Archives de l'Art français, 1852, t.1, p.94-13 et GRANDMAISON, Charles de. Documents inédits pour servir à l'histoire des arts en Touraine. Paris : Dumoulin, 1870, p. 44.

27. - Sa seule réalisation pour la reine Anne connue à ce jour est le tombeau de Charles VIII, conservé à Saint-Denis jusqu'à la Révolution et aujourd'hui disparu, mais documenté par différentes descriptions et relevés du xvIII siècle. Sur les réalisations de Guido Mazzoni lors de son séjour en France, voir VERDON, Timothy. «Guido Mazzoni in Francia : nuovi contributi ». Mitteilungen des Kunsthistorischen Institutes in Florenz, 1990, t. XXXIV, p. 139-164. Signalons aussi que l'activité de Guido Mazzoni en tant que peintre reste à ce jour mal documentée. Certains ont pu lui attribuer les peintures murales de la chapelle de l'hôtel de Cluny (SCAILLEREZ, Cécile. « Les peintures de la chapelle de l'hôtel de Cluny ». Dans CRÉPIN-LEBLOND, Thierry, BOS, Agnès, DECTOT, Xavier (dir.). L'Art des frères d'Amboise. Les chapelles de l'hôtel de Cluny et du château de Gaillon. Cat. exp., Paris, musée national du Moyen Âge/Écouen, musée national de la Renaissance, 3 octobre 2007-14 janvier 2008. Paris : Réunion des musées nationaux, 2007, p. 43-47). L'artiste est néanmoins bien qualifié de "peintre » lorsque la Ville de Paris s'adresse à lui pour réaliser le patron de la nef offerte à la reine pour son entrée de 1504 (HAMON, Étienne. Une Capitale flamboyante. La création monumentale à Paris autour de 1500. Paris : Picard, 2011, p. 133).

28. - Il n'y a pas lieu de développer ce point ici. Signalons seulement que l'étude des déplacements d'Anne de Bretagne confirme qu'elle fit du Plessis sa résidence principale, n'allant à Amboise qu'occasionnellement, pour y rejoindre le roi, tandis que Charles VIII résidait de préférence à Amboise. Voir VRAND, Caroline. Les Collections d'art d'Anne de Bretagne, 2016. Op. cit. 29. - Voir HALASZ CSIBA, Eva. Art. cit. L'auteur mentionne d'ailleurs la commande de 1496. 30. - Si l'on s'en tient à ce que nous apprend la comptabilité royale, les soubassements commandés par Anne ne sont pas en cuir doré mais uniquement en cuir peint. Sur la technique du cuir doré, qui se diffuse en Italie dans le courant du xvi siècle, voir THORNTON, Peter. The Italian Renaissance Interior. 1400-1600. Londres: Weidenfeld and Nicolson, 1991, p. 85-86. L'auteur mentionne d'ailleurs la commande de 1496 d'Anne de Bretagne et indique qu'il ne s'agit 
probablement pas de cuir peint. Je tiens également à remercier Muriel Barbier pour nos échanges sur les cuirs peints et pour ses orientations bibliographiques.

31. - Ferdinand $\mathrm{I}^{\mathrm{er}}$ de Naples (1458-1494) est le fils bâtard d'Alphonse V, roi d'Aragon (1416-1458), ce dernier étant l'oncle de Ferdinand II, roi de Castille (1474-1504) et d'Aragon (1479-1516), puis roi de Naples (1503-1516).

32. - AN, KK 83, fo 32.

33. - BnF, ms fr. 22335, p. 50-51 et 129 pour 1500 et p. 249-250 pour 1507.

34. - Signalons néanmoins la présence de quelques rares ensembles de lits brodés répertoriés à Amboise en 1494. BnF, fr. 22335, p. 161-162.

35. - FARCY, Louis de. Op. cit., p. 38. Pour autant, la présence de «fils d'or de Chypre », technique de traitement de l'or particulièrement réputée dans laquelle la ville de Gênes s'était spécialisée, autorise aussi à supposer que le roi de France a pu profiter de son passage dans le port ligure, à la fin de l'année 1499, pour y commander ces ciels.

36. - AN, KK 86, fo 61.

37. - Pour les liens entre Anne de Bretagne et Isabelle d'Este, voir BASCHET, Armand. «Quelques lettres missives extraites des Archives de la Maison de Gonzague ». Société de l'histoire de Francenotices et documents, 1884, p. 300 et BROWN, Clifford M. « Una immagine di Nostra Donna, Lorenzo Costa's Holy Family for Ann of Brittany ». Dans Cultura figurativa ferrarese fra XV et XVI secolo. In Memoria di Giacomo Bargellesi. Venise : Corbo e Fiore, 1981, p. 115-128.

38. - BnF, ms fr. 22335, p. 137. Pour une étude plus complète de ce lit et sur les échanges entre les deux femmes, nous renvoyons à notre contribution « Venise en Val de Loire : les verres vénitiens d'Anne de Bretagne ». Dans BRESC-BAUTIER, Geneviève, CRÉPIN-LEBLOND, Thierry, TABURETDELAHAYE, Élisabeth (dir.). La France et l'Europe autour de 1500. Croisements et échanges artistiques. Actes du colloque, Paris, École du Louvre, musée d'Écouen, 9-11 décembre 2010. Paris : École du Louvre, 2015, p. 25-33. Sur ce lit, voir également THIRION, Jacques et CRÉPIN-LEBLOND, Thierry (dir.). Op. cit., p. 21-22.

39. - Voir notamment l'enluminure réalisée par Jean Bourdichon au folio 1 des Épitres royaux et qui représente Anne de Bretagne écrivant une lettre au roi (Saint-Pétersbourg, Bibliothèque nationale de Russie, Fr Fv XIV 8).

\section{RÉSUMÉS}

La comptabilité et les inventaires royaux de Charles VIII (1483-1498), Louis XII (1498-1515) et Anne de Bretagne (1491-1514) livrent quantité d'informations sur l'aspect et la place du lit au sein des résidences royales autour de 1500. À cette époque, les éléments en bois s'effacent largement au profit des étoffes qui les recouvrent. Les matières, les couleurs et les sujets iconographiques varient en fonction de l'emplacement des lits et de leur destination. Les sources décrivent de façon assez précise certains lits dressés dans les châteaux royaux, tels ceux que Charles VIII fit réaliser pour sa résidence du Plessis-lès-Tours (La Riche, Indre-et-Loire) ou ceux qu'Anne de Bretagne commanda lorsqu'elle rejoignit le Val de Loire en tant que reine de France. Ces différentes commandes montrent combien les lits constituaient un élément primordial de l'ameublement et des collections royales. Indispensables à l'aménagement ordinaire des logis, les lits s'imposent en définitive comme des objets de prestige, voire de pouvoir. 
The royal account books and inventories of Charles VIII (1483-1498), Louis XII (1498-1515) and Anne of Brittany (Anne de Bretagne, 1491-1514) provide much information about the appearance and the place of the bed in royal residences around the year 1500. During this period, the bed's wooden structural elements came to be hidden by the fabrics which covered them. Materials, colours and decorative iconographical subjects varied according to the bed's place and its destination. The sources give precise descriptions of certain beds installed in royal palaces, such as the bed made for Charles VIII for his residence of Plessis-lès-Tours (at La Riche, Indre-et-Loire) or the beds made for Anne de Bretagne when she went to the Loire valley as Queen of France. The commissions for the construction of these beds show how they represented an essential element of royal collections and furniture. They were indispensable items for the day-to-day organisation of the royal household, but also came to be objects of prestige and even of power.

\section{INDEX}

Keywords : bed, furniture, royal collections, art objects, royal residences, tapestry, embroidery, silks, Anne of Brittany (Anne de Bretagne), Charles VIII, Louis XII, Isabelle d'Este, Renaissance, Middle Ages, Plessis-lès-Tours Chateau, Italian wars

Mots-clés : lit, ameublement, collections royales, objets d'art, logis royaux, tapisserie, broderie, soierie, Anne de Bretagne, Charles VIII, Louis XII, Isabelle d'Este, Renaissance, Moyen Âge, château du Plessis-lès-Tours, guerres d'Italie

\section{AUTEUR}

\section{CAROLINE VRAND}

Archiviste paléographe, docteur en histoire de l'art, conservateur du patrimoine ; responsable des estampes des $\mathrm{Xv}^{\mathrm{e}}$ et $\mathrm{XVI}^{\mathrm{e}}$ siècles au département des Estampes et de la Photographie de la Bibliothèque nationale de France carolinevrand@gmail.com 